Methods: A new word definition task was designed to overcome the ceiling effect. It was developed for elucidating semantic disorganization in word comprehension across grammatical categories (ie nouns, verbs and adjectives) and administered to 32 patients with schizophrenia, 28 patients with bipolar disorder and 32 matched healthy controls.

Results: A $3 \times 2 \times 3 \times 2$ repeated-measures ANOVA indicated that while both patient groups illustrated semantic processing deficits when compared with healthy controls, word comprehension across grammatical categories was comparable despite diagnosis, as was the influence of word frequency. All subject groups were better at defining adjectives compared with nouns and verbs, and comprehension increased for high-frequency words.

Conclusions: Despite the semantic processing deficits illustrated by both patient groups, processing of grammatical categories was not differentiated by the presence of psychosis. Additional research into the role of encoding, word organization, and specific basis for variation in the processing of adjectives warrants further research.

\section{The response to sulpiride in major depression before and after cognitive behavioural therapy: D2 receptor function}

\section{CJ Bell, S Bhikka, R Porter, C Frampton, J Carter, PR Joyce}

Department of Psychological Medicine, Christchurch School of Medicine and Health Sciences, Christchurch, New Zealand

Background: Previous studies of D2 receptor function in depressed patients have shown mixed results, with some (but not all) reporting increased sensitivity/ upregulation of D2 receptors in untreated depression, while others report that effective treatment results in increased sensitivity/upregulation.

Methods: D2 receptor function was assessed in 24 patients with major depression before and 16 patients after 16 weeks of treatment with cognitive behavioural therapy (CBT) using a challenge with a selective D2 antagonist, sulpiride. Four hundred milligrams of sulpiride was administered orally on two test days and response measured in two different dopaminergic pathways: the change in prolactin secretion (tuberoinfundibular pathway) and changes in self-rating scale measures of mood (VAS, POMS), anxiety (STAI) and pleasure (SHPS) (mesocorticolimbic pathway).

Results: There was no significant difference in the prolactin response to sulpiride before and after treatment $(z=-1.4, P=0.156)$. On both test days, sulpiride led to an improvement in mood (VAS and
POMS scales). After CBT, this effect was significantly reduced as measured by the POMS scale $(t=-2.3$, $P=0.038$ ) but unchanged on the VAS scale. Although patients exhibited significant clinical improvement after treatment (as measured by percentage improvement in HDRS score), there was no correlation between response to $\mathrm{CBT}$ and changes in response to sulpiride in either pathway.

Conclusions: No change in tuberoinfundibular D2 receptor function was detected following CBT. A change in mesocorticolimbic D2 receptor function was detected; however, no relationship between changes in D2 receptor sensitivity and clinical response to CBT was evident in this group of depressed patients.

\section{The Clinical Global Impressions Scale Modified for Substance Use (CGI-SU) in patients with bipolar disorder}

\section{Bernardo', M Berk', S Dodd', K Lu², 0 Dean', I Schapkaitz², S Jeavons ${ }^{2}$}

${ }^{1} T$ The University of Melbourne, Melbourne, Australia; and ${ }^{2}$ Mental Health Research Institute, Melbourne, Australia

Background: Bipolar disorder is a chronic illness that is commonly associated with comorbid substance use. The development of the Clinical Global Impressions Scale Modified for Substance Use (CGI-SU) was to produce a simple, quick and easy-to-administer assessment tool. It was used in a randomized placebocontrolled trial of $\mathrm{N}$-acetylcysteine (NAC) in bipolar disorder. It was hypothesized that NAC would improve the outcomes in bipolar disorder and ultimately in comorbid substance use.

Methods: The study focused on the CGI-SU, which is a newly developed assessment tool based on the original CGI for improvements. The new scale is intended for use in routine clinical practice to monitor improvements in a patient's comorbid substance use. It was administered to participants $(n=75)$ in the NAC in bipolar disorder trial. The CGI-SU scale investigates changes in six items, including ethanol, caffeine, nicotine, delta-9-tetrahydrocannabinol and two additional items depending on the participants' use of substances.

Results: About $78.7 \%$ were using ethanol, $92 \%$ caffeine, $45.3 \%$ nicotine and $7.9 \%$ delta-9-tetrahydrocannabinol. These participants' use of substances permitted us to monitor the improvements of their substance use over the trial period.

Conclusions: The simplicity and brevity of the CGISU make it a valuable outcome measure. It enables the monitoring of the improvements or lack of improvements made by patients with bipolar disorder and 
comorbid substance use in observational studies and clinical practice.

\section{The impact of accommodation status on the hospitalization of mentally ill patients}

\author{
A Bonett', S Tahtalian ${ }^{2,3}$, B Hayward' ', J Kulkarni' ${ }^{2,3}$, \\ Y Hollander ${ }^{1}$ \\ 'Alfred Hospital Psychiatry Department; ${ }^{2}$ Alfred Psychiatry Research Centre; and \\ ${ }^{3}$ Monash University, Melbourne, Australia
}

Background: The importance of suitable and stable housing has long been recognized for people suffering mental illness. In recent years, there has been a steady decrease in the availability of housing options for this group of people. Anecdotal evidence suggests that this is now impacting on their length of hospitalization and health outcomes.

Method: Patients were enrolled over a 3-month period during their admission to the Alfred Hospital psychiatric ward. In-patient social workers collected information for every consecutive admission regarding patient's current accommodation situation and their attempt to secure appropriate housing for these patients. Demographic information was also collected. Consultant psychiatrists measured symptom severity using the Brief Psychiatric Rating Scale.

Results: Data presented will include a summary of the appropriateness of the current housing situation of participants at admission, the accommodation options available for discharge, the time taken to secure the accommodation, the time spent in hospital after medical clearance for discharge and the length of hospitalization. An analysis will assess whether patients who are considered to be in unstable accommodation are experiencing longer hospital stays as compared with those in stable housing.

Conclusions: The results will examine how the decreases in accommodation options are impacting on the use of hospital resources and patient outcomes. This information is imperative given the limited inpatient psychiatric resources available.

\section{Keep the heart in mind: the interplay of heart and brain activity over the life span}

\section{P Boord', L Williams', E Gordon², C Rennie'}

'Brain Dynamics Centre, Westmead Millenium Institute, Westmead Hospital and the University of Sydney, Australia; and 'Brain Resource Company, Sydney, Australia
Background: Affective disorders are associated with both visceral and neurophysiological changes, but few studies report simultaneous measurement and analysis of these systems. We used an integrative neuroscience approach to explore relationships between heart and brain activity for healthy controls, as a benchmark for studying the interplay of these systems in affective disorders.

Methods: Participants were recruited in collaboration with the Brain Resource International Database (www. brainresource.com). Simultaneous EEG and ECG were recorded from 2092 healthy individuals while they rested with their eyes open. EEG power was calculated in standard frequency bands, averaged across sites and correlated with average heart rate for each decade of the life span.

Results: Heart rate was significantly correlated with EEG power in young people (10-20 years old) but was less evident in people outside this age range. In this age group, heart rate and EEG power had a positive correlation across all frequency bands (delta, theta, alpha, beta $r=+0.25,+0.20,+0.14,+0.19$, respectively; $n=675, P<0.001$ ).

Conclusions: This study shows the imperative of using an integrative neuroscience approach in the study of brain function and dysfunction. Without taking account of the manifold influences on brain function, neurophysiological studies might be confounded by variance in these factors. Simultaneous measurement across systems can also disclose their interaction in health and disease. The observed change in interplay between the heart and brain over age might prove an important factor in the understanding of affective disorders.

\section{Reduced fMRI activity in response to salient stimuli in first-episode schizophrenia}

\section{K Brown', D Alexander², P Boord', P Das 1,3, G Flynn', C Galletly ${ }^{5}$, E Gordon ${ }^{1,2}$, A Harris ${ }^{1,6}$, TJ Whitford', W Wong ${ }^{4}$, L Williams ${ }^{1,6}$}

'Brain Dynamics Centre, Westmead Millenium Institute, Westmead Hospital and the University of Sydney, Australia; ${ }^{2 B r a i n}$ Resource Company; ${ }^{3}$ Neuroscience Institute of Schizophrenia and Allied Disorders (NISAD); "Liverpool Hospital, Early Psychosis Intervention Program; ${ }^{5}$ University of Adelaide; and ${ }^{6}$ The University of Sydney, Australia

Background: Our integrative neuroscience model of schizophrenia highlights the lack of coordinated neural activity required for effective processing of salient and task-relevant stimuli. The 'auditory oddball' task taps the fundamental mechanisms of selecting and responding to salient stimuli, and disturbances on this task are a trait-like marker for schizophrenia. The objective of 